\title{
Do teashirt family genes specify trunk identity? Insights from the single tiptop/teashirt homolog of Tribolium castaneum
}

\author{
Teresa D. Shippy • Yoshinori Tomoyasu • \\ Wensheng Nie - Susan J. Brown • Robin E. Denell
}

Received: 3 January 2008 / Accepted: 12 February 2008

(C) The Author(s) 2008

\begin{abstract}
The Drosophila teashirt gene acts in concert with the homeotic selector (Hox) genes to specify trunk (thorax and abdomen) identity. There has been speculation that this trunk-specifying function might be very ancient, dating back to the common ancestor of insects and vertebrates. However, other evidence suggests that the role of teashirt in trunk identity is not well conserved even within the Insecta. To address this issue, we have analyzed the function of Tc-tiotsh, the lone teashirt family member in the red flour beetle, Tribolium castaneum. Although Tc-tiotsh is important for aspects of both embryonic and imaginal development including some trunk features, we find no evidence that it acts as a trunk identity gene. We discuss this finding in the context of recent insights into the evolution and function of the Drosophila teashirt family genes.
\end{abstract}

Keywords Tribolium $\cdot$ Teashirt · Tiptop · Trunk $\cdot$ Homeotic

Electronic supplementary material The online version of this article (doi:10.1007/s00427-008-0212-5) contains supplementary material, which is available to authorized users.

Communicated by S. Roth

T. D. Shippy $(\bowtie) \cdot$ Y. Tomoyasu $\cdot$ W. Nie $\cdot$ S. J. Brown $\cdot$

R. E. Denell

Division of Biology, Kansas State University,

Manhattan, KS 66506, USA

e-mail: tshippy@ksu.edu

Present address:

W. Nie

Fibrogen,

225 Gateway Boulevard,

South San Francisco, CA 94080, USA

\section{Introduction}

Homeotic genes are responsible for defining regional identity, and mutations in these genes often transform one body part into another. The best characterized homeotic genes are the Hox genes, which have been identified in representatives of most animal phyla (reviewed by Martindale 2005) and have been extensively studied in several organisms. However, some non-Hox genes have also been classified as homeotic genes based on their mutant phenotypes. One such gene encodes the zinc finger transcription factor Teashirt (Fasano et al. 1991). Loss-of-function (LOF) teashirt (tsh) mutations are reported to cause partial transformation of the larval prothorax to a labial identity due to ectopic expression of the Hox gene Sex combs reduced (Fasano et al. 1991; Roder et al. 1992). The trunk (thoracic and abdominal) segments of $t s h^{-}$larvae are shorter than normal and the denticle belts (a trunk-specific feature) are severely disorganized. Moreover, patches of sclerotic cuticle, which have been interpreted as head structures, form between the remaining denticle belts (Fasano et al. 1991). Conversely, ectopic expression of tsh results in the development of denticle belts in the head (de Zulueta et al. 1994). On the basis of these phenotypes, tsh has been suggested to function as a specifier of trunk vs head identity (Roder et al. 1992).

tsh is believed to act in parallel with the trunk Hox genes (Scr, Antennapedia (Antp), Ultrabithorax (Ubx), abdominal-A (abd-A), and Abdominal-B (Abd-B)) to determine segmental identity (de Zulueta et al. 1994; Roder et al. 1992). When all of these Hox genes are inactivated, trunk segments develop with what has been interpreted as a mixed head/trunk identity. That is, sclerotized tissue develops in the posterior compartment of each trunk segment, whereas denticle belts (a trunk-specific feature) still develop in the anterior compartment (Sato et al. 1985; 
Struhl 1983). When tsh function is also eliminated, the trunk segments are more severely affected in that denticle belts disappear and additional head-like cuticle is observed (Roder et al. 1992).

Recent data suggests that $t s h$ may play an even greater role in trunk development than was previously recognized. In the absence of tsh function, its paralog tiptop (tio) is ectopically expressed in the trunk region (Laugier et al. 2005). When tio and $t s h$ functions are both absent, the trunk denticles are even more severely affected than in mutants that lack only tsh, suggesting that tio can partially substitute for $t s h$ and reduce the effects of $t s h$ mutations.

Robertson and coworkers (2004) proposed the existence of a system of zinc-finger proteins that divides the Drosophila embryo into sections and limits the functional domains of Hox genes. They suggest that within this system tsh specifies the trunk region, while disconnected (disco) specifies the gnathal region. In the absence of tsh, disco (as well as its paralog disco-related) is ectopically expressed in the trunk, and there is some evidence that ectopic disco expression is responsible for at least some features of $t s h$ mutants (e.g., loss of denticles and abnormal sensory neurons) that are thought to represent a switch to head/gnathal identity (Robertson et al. 2004).

Manfroid et al. (2004) speculated that the trunkspecifying function of tsh family members might be conserved in vertebrates, as $t s h$ genes from mice are expressed in the trunk region and can also rescue the phenotype of Drosophila tsh mutants when ectopically expressed. However, such conjecture requires a rather large phylogenetic leap, as it is not clear that the role of tsh in trunk specification is conserved even in other insects. tsh family genes of the firebrat, Thermobia domestica, and the milkweed bug, Oncopeltus fasciatus, are expressed predominantly in the gnathos and thorax, arguing against a function in trunk specification (Herke et al. 2005; Peterson et al. 1999). Furthermore, when Herke et al. (2005) knocked down the function of the Oncopeltus tsh family gene by RNA interference (RNAi), they observed transformations of distal leg to antenna, but did not report any changes in trunk identity. Finally, our previous studies suggest that genetic specification of trunk identity in the red flour beetle, Tribolium castaneum, differs significantly from that in Drosophila. Removal of trunk Hox genes alone is sufficient to produce dramatic transformation of trunk to head identity (Brown et al. 2002). Antennae develop on all gnathal and trunk segments of embryos homozygous for a deficiency that removes the Tribolium orthologs of Deformed (Dfd), Scr, Antp, Ubx, and abd-A (Stuart et al. 1991). Thus, there is apparently no need to invoke the function of another gene for trunk identity. Peterson et al. (1999) predicted that either the Tribolium tsh gene has no function in trunk specification or that the expression of tsh is controlled by
Hox genes. Taken together, these observations suggest that the trunk-specifying role of tsh observed in Drosophila might not be conserved in other insects.

To better understand the evolution of $t s h$ family gene function, we cloned and characterized a tsh homolog from Tribolium. This gene, Tc-tiotsh, is the sole member of this family in the Tribolium genome. It has a broad embryonic expression domain, encompassing not only the trunk region but much of the head as well. RNAi experiments revealed that $T c$-tiotsh functions in several embryonic and imaginal tissues, but they did not suggest a conserved function in trunk specification. We discuss these results and reexamine the classification of tsh as a trunk-specifying gene in Drosophila.

\section{Materials and methods}

\section{cDNA cloning}

A genomic fragment of the Tc-tiotsh gene was amplified using degenerate primers based on the conserved amino acid motifs VWLGKG and CKVCDK (first round) and ILKCMW and CKVCDK (second round). Additional, overlapping fragments were identified by RACE, using the $5^{\prime}$ and 3' RACE Systems (GibcoBRL), by polymerase chain reaction (PCR) screening a Tribolium castaneum cDNA library as described previously (Nie et al. 2001), or by inverse PCR (Ochman et al. 1988). To confirm that these overlapping fragments represent a contiguous sequence, primers were designed near the ends of the putative contig, and reverse transcriptase (RT)-PCR was performed using total embryonic RNA ( $0-30 \mathrm{~h}$ of development) as template. The resulting PCR product was cloned and sequenced (GenBank accession number AF356647).

\section{Sequence analysis}

The nucleotide contig for Tc-tiotsh was assembled using the Seqman module of Lasergene (DNASTAR). To identify putative $t s h$ family members in other genomes, we performed tBLASTn searches using Drosophila melanogaster Tsh and Tio as query sequences. Multiple alignments were created with the AlignX module of Vector NTI 8 (InforMax). Minor manual adjustments were performed to maximize the alignments. The final output of the multiple alignment was created using BOXSHADE (available at http://www.ch.embnet.org/software/BOX_form. html). Phylogenetic analyses were performed using $M E G A$ version 3.1 (Kumar et al. 2004) or TreePuzzle (http:// bioweb.pasteur.fr/seqanal/interfaces/Puzzle.html), and TreeView (http://taxonomy.zoology.gla.ac.uk/rod/treeview.html) was used to visualize the output. 
In situ hybridization and immunostaining of embryos

A digoxigenin-labeled fragment of Tc-tiotsh was hybridized to whole-mount wild-type (Georgia-1) embryos as previously described (Brown et al. 1994b). Embryos were secondarily immunostained with either $\alpha$-Invected antibody (4D9) (Patel et al. 1989) or with $\alpha$-Even-skipped antibody (2B8) (Patel et al. 1994), which cross-react with Tc-Engrailed and Tc-Even-skipped, respectively. Tc-spalt (Tc-sal) expression was assayed using the probe described by Tomoyasu et al. (2005).

\section{RNA interference (RNAi)}

dsRNA of two different, non-overlapping fragments of $T c$ tiotsh was synthesized using the PCR template method described by Kennerdell and Carthew (2000). Briefly, primers were chosen to amplify a fragment of Tc-tiotsh (bases 13581786 or 1848-2581 of GenBank sequence AF356647) and the T7 promoter sequence (TAATACGACTCACTATAGG) was added to the $5^{\prime}$ end of each primer. The resulting primers were used to amplify template for RNA synthesis. The resulting product was purified and $1 \mu \mathrm{g}$ used as template for RNA synthesis using the Megascript T7 High Yield Transcription kit (Ambion).

For embryonic RNAi, 0-2 h eggs were collected from wild-type beetles (GA-1) and injected with dsRNA (2-4 $\mu \mathrm{g} /$ $\mu \mathrm{l})$ as previously described by Brown et al. (1999). After several days, hatched larvae and unhatched eggs were treated with lactic acid/ethanol. Additional larvae were documented by SEM as described by Curtis et al. (2001).

Injection into non-virgin adult females was performed by manually opening the elytra and wings of etheranesthetized beetles and injecting $2 \mu \mathrm{g} / \mu \mathrm{l}$ dsRNA into the dorsal abdomen. Larval RNAi was performed as described by Tomoyasu and Denell (2004).

\section{Results}

Tribolium has a single teashirt family gene

We cloned a member of the tsh gene family from the red flour beetle, Tribolium castaneum (see Materials and methods for details). Comparison of the cDNA sequence to the recently completed Tribolium genome sequence revealed three exons distributed over about $90 \mathrm{~kb}$ (Fig. 1). Most of this distance can be attributed to an approximately 86-kb intron between the first and second exons.

Only three full-length Tsh family proteins have been described from insects: the Drosophila melanogaster paralogs Tsh and Tio, and the single member of the Tsh family in Anopheles gambiae, Ag-TioTsh (Fasano et al.
1991; Laugier et al. 2005). In addition, small fragments of tsh family genes have been amplified from several other insects (Herke et al. 2005; Peterson et al. 1999). Two conclusions have been drawn from these limited data. First, the two Drosophila genes seem to be the result of a recent duplication in the Drosophila lineage. Second, all of the Tsh family members from other insects appear to be more similar in sequence to Tio than to Tsh. In this paper, we present data that support and greatly strengthen these conclusions.

We performed a tBLASTn search of several recently sequenced insect genomes for $t$ sh family members (Table 1 ). The Tribolium castaneum gene that we cloned is the only member of the $t s h$ family present in the genome sequence of this insect. Likewise, the Apis mellifera (honeybee) genome contains only a single member of this gene family. In contrast, all of the additional Drosophila species we searched (D. pseudoobscura, D. simulans, D. yakuba and D. virilis) have orthologs of both tio and tsh. Consistent with the situation in D. melanogaster (Laugier et al 2005), tio and tsh are closely linked (less than $250 \mathrm{~kb}$ apart) in all of the Drosophila species examined, suggesting that these genes likely arose from a relatively recent tandem duplication event.

As the Tribolium gene is related to both tio and tsh, we have named it Tc-tiotsh, after the convention established by Laugier et al. (2005) for the single Anopheles gene. We compared the predicted Tc-TioTsh protein to the other insect Tsh family members for which full-length predicted proteins are available. (Although, we were able to identify a tsh family gene in Apis, the $5^{\prime}$ portion of the transcription unit was apparently missing from the genome sequence.) Like tio and Ag-tiotsh (Laugier et al. 2005), Tc-tiotsh encodes four zinc fingers (Fig. 1), three of which are clustered near the middle of the protein (Fig. 2a), and one that is located near the $\mathrm{C}$ terminus (Fig. 2b). Besides the zinc finger regions, several additional short regions of sequence identity (shaded in Fig. 2 and data not shown) are present in all of the full-length predicted proteins. The three dipteran proteins are highly similar at their $\mathrm{N}$ termini, while Tio, Ag-TioTsh, and Tc-TioTsh share a C-terminal motif (in addition to the fourth zinc finger) that is absent in Tsh. Regions shared between the beetle and dipteran proteins have been conserved over a long phylogenetic distance, suggesting that they likely have important functions.

To better understand the evolutionary history of the $t s h$ gene family, we have performed phylogenetic analyses using conserved portions of the zinc-finger region (underlined in Fig. 2) from the full-length insect Tsh family proteins that are currently available (Drosophila, Tribolium, and Anopheles). Both maximum likelihood (Fig. 3) and neighbor-joining (data not shown) methods produce trees in 


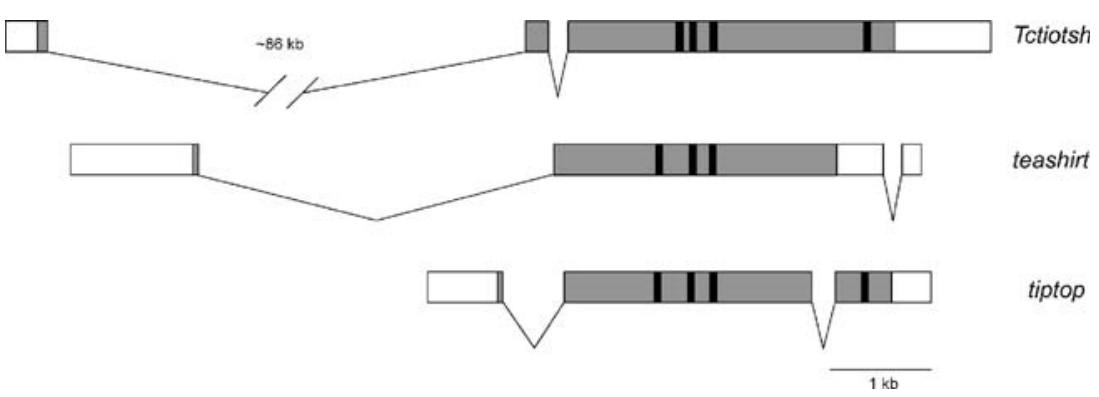

Fig. 1 Gene structure of the Tc-tiotsh gene and its Drosophila homologs. Exons are denoted by boxes and introns by thin lines. The coding region is shaded gray and zinc finger-encoding motifs are shown in black

which the two Drosophila proteins cluster together, suggesting these genes were produced by duplication after the divergence of the mosquito and fly lineages. The branch lengths of the Drosophila proteins are much longer than those of Anopheles and Tribolium, suggesting rapid divergence of both genes after duplication.

The mouse and human genomes each have three tsh family members (Caubit et al. 2000; Manfroid et al. 2004), which are much more similar to one another than they are to any of the insect proteins. Interestingly, vertebrate Tsh family proteins share an N-terminal motif (MPRRKQ) with the Tribolium protein (Fig. 2c), which is distinct from the $\mathrm{N}$-terminal motif shared by the dipteran family members (Laugier et al. 2005). The conservation of this sequence in an insect and vertebrates suggests it has an important function and that it was probably present in the common ancestor of protostomes and deuterostomes.

Taken together, these comparisons of tsh family members indicate that the ancestor of protostomes and deuterostomes had a single $t s h$ family gene. We were unable to identify any tsh family members in the genomes of two non-bilaterian metazoans: the placozoan Trichoplax adhaerens (http://genome.jgi-psf.org/Triad1/Triad1.home.html) and the cnidarian Nematostella vectensis (Putnam et al. 2007), suggesting this gene family may have arisen after the bilaterian/non-bilaterian split. Within the vertebrate lineage, duplication events (perhaps in association with whole genome duplications) produced at least three paralogs. A duplication in the Drosophila lineage (after divergence from the mosquito lineage) resulted in the $t s h$ and tio paralogs.

Tc-tiotsh embryonic expression is not limited to the trunk

We examined the embryonic expression pattern of Tc-tiotsh (Fig. 4) by in situ hybridization, using the posterior compartment marker Engrailed (Brown et al. 1994a; Patel et al. 1989) to determine the stage of embryonic development (segments are added sequentially) and provide a reference for the register of Tc-tiotsh expression. Tc-tiotsh expression develops in a dynamic, pair rule-like manner (Fig. S1 and S2), but eventually encompasses not only the trunk segments (thorax and abdomen), but also the gnathal segments and the labrum and intercalary segment in the procephalon (Fig. 4a). Tc-tiotsh expression in the thorax and the first abdominal segment appears somewhat stronger than in other regions (Fig. 4b). As appendages develop, Tc-tiotsh is expressed in the proximal portion of each leg (Fig. 4d).

After germ-band elongation, Tc-tiotsh is expressed in the posterior region of the embryo. Initially, expression is seen in a patch on either side of the ventral midline (Fig. 4c), and later in six buds that extend from the invaginating hindgut (Fig. 4e). These structures are the primordia of the Malpighian tubules; they also express Tc-dachsund (Prpic et al. 2001) and Tc-Cut (data not shown).

Table 1 Teashirt family genes in sequenced insect genomes

\begin{tabular}{llll}
\hline Organism & Order & tsh family genes & Genome Reference \\
\hline Tribolium castaneum & Coleoptera & 1 & The Tribolium Genome Sequencing Consortium 2008 \\
Apis mellifera & Hymenoptera & 1 & The Honeybee Genome Sequencing Consortium 2006 \\
Anopheles gambiae & Diptera & 1 & Holt et al. 2002 \\
Drosophila simulans & Diptera & 2 (tio and tsh) & Drosophila 12 Genomes Consortium 2007 \\
Drosophila yakuba & Diptera & 2 (tio and tsh) & Drosophila 12 Genomes Consortium 2007 \\
Drosophila pseudoobscura & Diptera & 2 (tio and $t$ sh) & Richards et al. 2005 \\
Drosophila virilis & Diptera & 2 (tio and tsh) & Drosophila 12 Genomes Consortium 2007
\end{tabular}

tBLASTn searches were used to determine the number of teashirt $(t s h)$ family genes in several assembled insect genomes. All Drosophila species examined had orthologs of both tsh and tiptop (tio), while the non-drosophilid species each contained only one tsh family member. 
Fig. 2 Multiple alignments of Teashirt family members. Amino acids identical or similar in at least two of the proteins are shaded black or gray, respectively. Tc Tribolium castaneum; Ag Anopheles gambiae, Dm Drosophila melanogaster; $\mathrm{Mm}$ Mus musculus; Dr Danio rerio. a Multiple alignment of the three clustered zinc fingers. Zinc fingers (ZF) 1-3 are indicated above the sequence. Regions underscored with asterisks were concatenated and subjected to phylogenetic analysis. b Multiple alignment of the $\mathrm{C}$-terminal zinc finger region. Zinc finger 4 is delimited above the alignment. Tsh lacks this zinc finger. c Distinct N-terminal motifs in dipteran Tsh family members (upper) and in Tribolium and vertebrates (lower) a

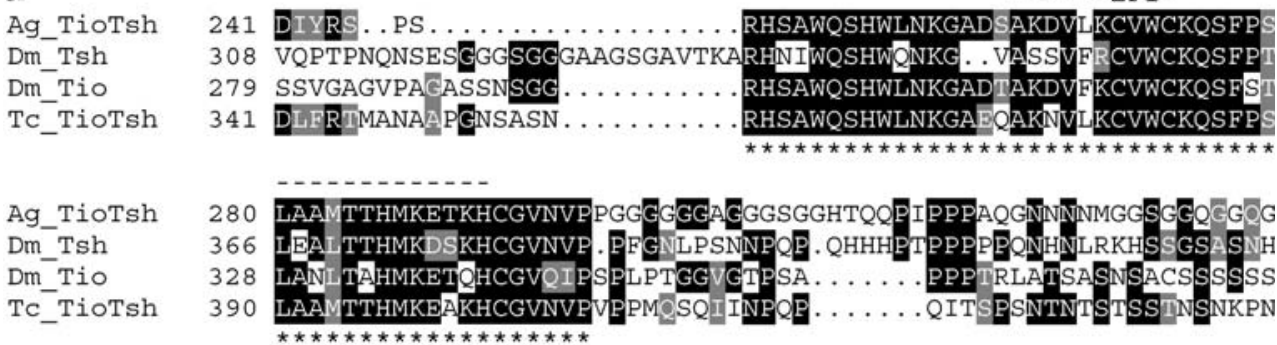

Ag_TioTsh

Dm_Tsh

Dm_Tio

Tc_TioTsh

Ag_TioTsh

Dm_Tsh

Dm-Tio

Tc_TioTsh

Ag_TioTsh

Dm_Tsh

Dm_Tio

Tc_TioTsh

b

Ag_TioTsh

Dm_Tsh

Dm Tio

Tc_TioTsh

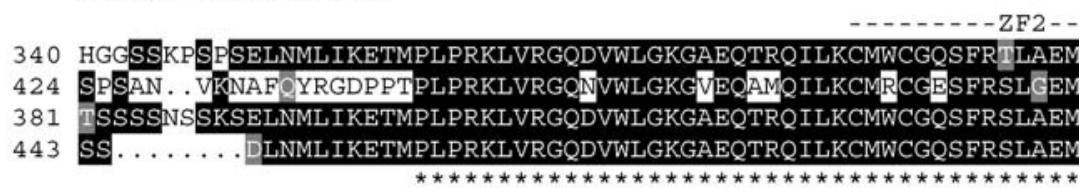

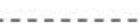

400 TAHMQQTQHYTNI ISQEQI ISWKSGGGANGGAG . GGAGGPASAAAAAAQTNSHVSAVLTC

482 TKHMQETQHYTNILSQEQSISIKSGNANAN . . . . . SDAKESHNSLSSEESRTLSAVLTC

441 TSHMQETQHYTNI ISQEQI ISWKSGDERERPTNTGVPSTS AAPSSPSCTAPSVSAVLTC 495 TSHMQQTQHYTNI I SQEQI ISWRSSDDAKG. . . GGGGSGSGNNQPPGGPSSHVSAVLTC

- - - - - - ZFB - - - - - - -

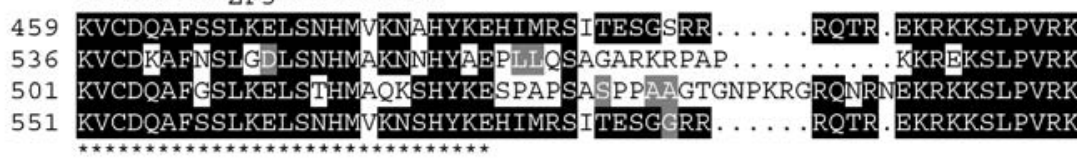

551 KVCDQAFSSLKELSNHMVKNSHYKEHIMRSITESGGRR . . . . . RQTR. EKRKKSLPVRK
928 ...G. TIKCSYCDTTFGSKGAYRHHLSKAHFVSDDVIPDPKVPARVVAPCPLPLPAAPSE

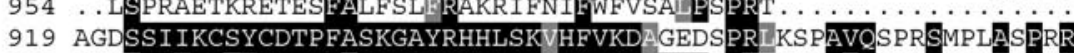
$1051 \ldots$. DSIMKCAFCDTPEISKGAYRHHLSKMHFVKDGVIPDPVALKSAQQGSSSSSEGVPLK

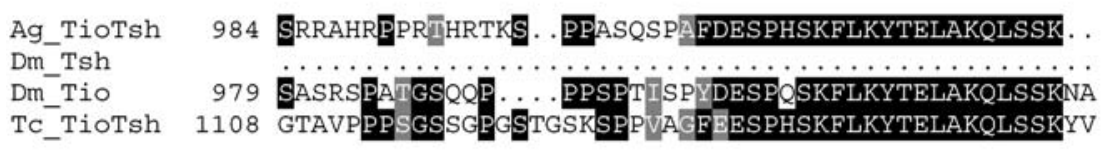

C

\begin{tabular}{lr|l} 
Ag_TioTsh & 1 & MLHEAVMLEIYRQAL \\
Dm_Teashirt & 1 & MLHEALMLEIYRQAL \\
Dm_Tiptop & 1 & MLHEAVMLEIYRQAL \\
Mm_tsh1 & 10 & MPRRKQQAPRR \\
Mm_tsh2 & 1 & MPRRKQQAPKR \\
Mm_tsh3 & 1 & MPRRKQQAPRR \\
Dr_tsh-1ike & 1 & MPRRKQQAPKR \\
Tc_Tiptop & 1 & MPRRKQDCPKR
\end{tabular}

spalt does not appear to repress Tc-tiotsh in Tribolium embryos

In Drosophila, the limits of tsh expression are controlled either directly or indirectly by spalt-major (salm). In a salm mutant, the $t s h$ expression domain expands both anteriorly and posteriorly (Roder et al. 1992). To assess whether the broader expression of Tc-tiotsh (compared to tsh) might reflect a difference in Tribolium spalt (Tc-sal) expression, we examined the embryonic expression pattern of the single Tribolium sal gene (Tomoyasu et al. 2005). We find that the Tc-sal expression domain (Fig. S3) significantly overlaps that of Tc-tiotsh, suggesting that Tc-sal does not repress $T c$ - tiotsh expression. It is possible that Tribolium has lost the regulatory interaction between these genes. Alternatively, regulation of $t s h$ by sal may have evolved more recently, after the divergence of these lineages.

Tc-tiotsh expression is reduced in embryos lacking Hox genes

In Drosophila, some aspects of tsh expression are regulated by the Hox genes (Roder et al. 1992). We have examined expression of Tc-tiotsh in embryos homozygous for $D f$ (HOMC), a deletion that removes most of the Tribolium Hox cluster. Tc-tiotsh expression is severely reduced in the ventral region of these embryos (Fig. 4f). These results 


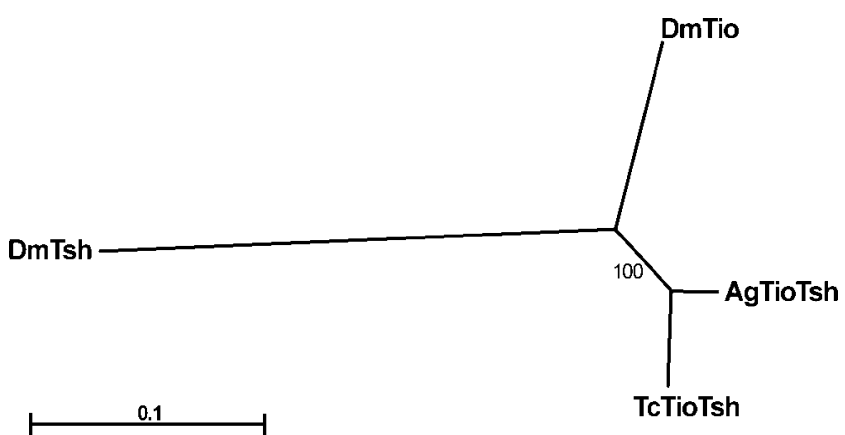

Fig. 3 Phylogenetic analysis of insect Teashirt family members. The two Drosophila paralogs cluster together in an unrooted tree produced by maximum likelihood analysis, suggesting that these genes resulted from a recent duplication in the Drosophila lineage. The bootstrap value of 100 indicates that the tree is very robust

suggest that reduction of Tc-tiotsh expression might contribute to the transformations seen in $D f(H O M C)$ embryos. However, our functional studies of Tc-tiotsh (presented below) reveal no evidence for this.

Tc-tiotsh functions in the embryonic labial and thoracic segments

We used RNAi to ascertain the probable LOF phenotype of Tc-tiotsh. Pupae injected with Tc-tiotsh dsRNA produced very few eggs upon reaching adulthood, and none of these progeny showed an abnormal phenotype. To circumvent the apparent effect of Tc-tiotsh on oogenesis, we injected $T c$ tiotsh dsRNA either directly into eggs or into the abdomens of non-virgin adult females (egg production from these females quickly tapers off, further indicating an effect on oogenesis). Injection of Tc-tiotsh dsRNA by either technique produced embryos with defects in the labial and thoracic segments (Fig. 5, Table 2, and data not shown). During normal Tribolium embryonic development, the two labial appendages fuse at the ventral midline and migrate anteriorly to a position between the maxillary appendages (Fig. 5a). In moderately affected Tc-tiotsh RNAi larvae (Fig. 5b), the labial appendages are smaller than normal, and in the most severely affected embryos (Fig. 5c) these appendages fail to fuse. In all of these cases, the abnormal labial appendages fail to migrate to their normal position between the maxillary palps. These abnormalities produce secondary effects on the orientation of the other head appendages.

Short legs were the most common feature seen in $T c$ tiotsh RNAi larvae, being present in all larvae with labial defects, as well as in some larvae with apparently normal labial appendages (Table 2). The reduction in leg length stems from effects on the proximal leg segments. The coxa and trochanter are reduced and apparently fused, and the femur is slightly shortened (Fig. 5e). This phenotype is similar to that observed in the adult legs of hypomorphic Drosophila tsh mutants (Erkner et al. 1999) and thus may indicate a conserved role for tsh family genes in proximal limb development.

We also noted that Tc-tiotsh RNAi larvae show a pronounced curvature of the head and thoracic segments, such that the head bends forward (Fig. 5e). This phenotype apparently results from reduction in length (anterior to posterior) of the ventral portions of the thoracic segments and posterior head (Fig. 5b,c,f).

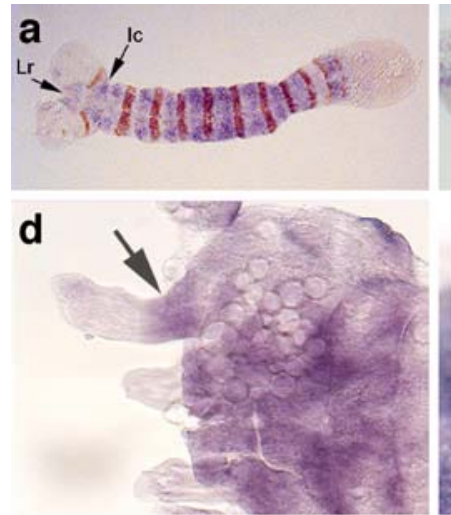

Fig. 4 Tc-tiotsh expression in Tribolium embryos. Engrailed expression is shown in brown, while Tc-tiotsh expression is in purple. Embryos are oriented with anterior to the left (except in panel $\mathbf{d}$ in which anterior is to the top). a During germband elongation, Tc-tiotsh is expressed in the gnathal and trunk segments as well as two procephalic regions (the intercalary segment $[I c]$ and the labrum $[L r])$. b When germband elongation is completed, Tc-tiotsh expression is strongest in the thoracic (T1-T3) and first abdominal (A1) segments. c Slightly later, strong
Tc-tiotsh expression appears in the posterior terminal region of the embryo (arrowhead). d Tc-tiotsh is expressed in only the proximal portion (arrow) of the developing legs. e In embryos undergoing germband retraction, Tc-tiotsh expression is present in the six Malpighian tubule primordia that evert from the hindgut. f In a $D f(H O M C)$ homozygote lacking most Hox genes, Tc-tiotsh expression in the segmented portion of the germband is greatly reduced. In contrast, expression in the Malpighian tubule primordia remains strong 

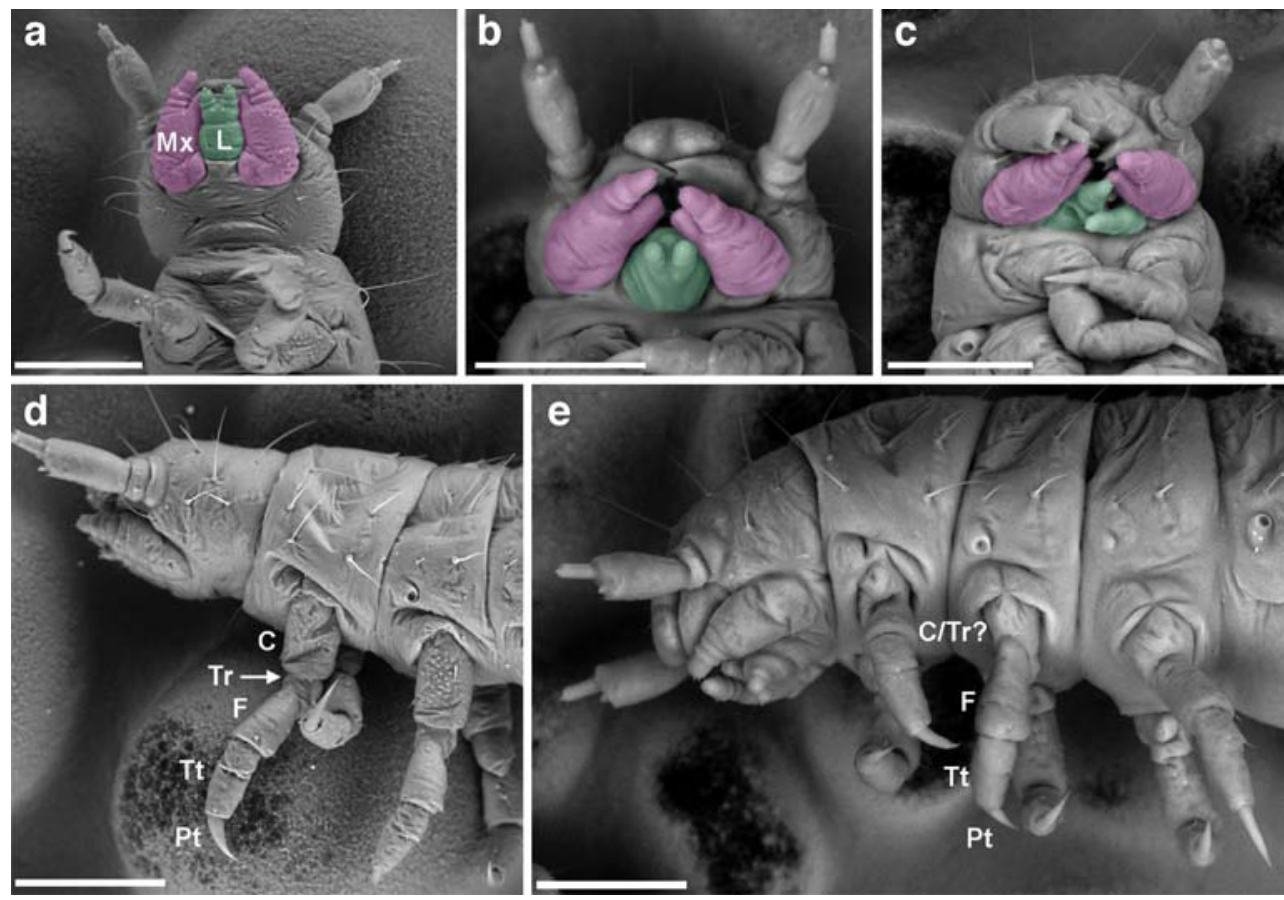

Fig. 5 Scanning electron micrographs of Tc-tiotsh RNAi larvae. For all panels, the scale bar represents 50um. a-c Ventral views of larval heads. Maxillary (pink) and labial (green) appendages have been shaded. d-e Lateral views with leg segments labeled as follows: coxa $(C)$, trochanter $(T r)$, femur $(F)$, tibiotarsus $(T t)$, and pretarsus $(P t)$. a Wild-type larval head showing the proper position of the maxillary $(M x)$ and labial $(L)$ appendages. b Moderately affected Tc-tiotsh RNAi

larva in which the labial appendages (especially the proximal region) are highly reduced. c Severely affected Tc-tiotsh RNAi larva in which the labial appendages have failed to fuse. Note that in both $\mathbf{b}$ and $\mathbf{c}$, the ventral, posterior region of the head (presumed to derive from the labial segment) is almost completely absent. d Wild-type larva. e $T c$ tiotsh RNAi larva with short legs. On each leg, the trochanter is presumably fused with the reduced coxa
Tc-tiotsh is important for proper development of adult trunk segments

We examined the role of Tc-tiotsh in adult development using larval RNAi (Tomoyasu and Denell 2004). The resulting adults display a number of defects in the thoracic and abdominal segments (Fig. 6). Most noticeably, these individuals have abnormalities of the $\mathrm{T} 2$ notum that prevent the elytra from closing properly (data not shown). We also observed mild defects in the proximal legs. In addition, adults resulting from Tc-tiotsh larval RNAi show abnormalities in many of the ventral sclerites (sternites) (see ElKifl 1953 for a description of wild-type structures). In the ventral prothorax (T1) of RNAi-treated individuals, the epimera, which are normally located posterior to the $\mathrm{T} 1$ coxae, are missing and the basisternum is reduced in size resulting in the exposure of membranous areas (Fig. 6b,d). In addition, the shape of the sternellum (the process

Table 2 Tc-tiotsh RNA interference cuticular phenotypes

\begin{tabular}{|c|c|c|c|c|}
\hline \multirow[t]{2}{*}{ dsRNA fragment } & \multicolumn{4}{|c|}{ Severity of Effect } \\
\hline & None & Mild & Moderate & Severe \\
\hline $1358-1786$ & $12 / 74(16.2 \%)$ & $4 / 74(5.4 \%)$ & $14 / 74(18.9 \%)$ & $44 / 74(59.5 \%)$ \\
\hline $1358-1786$ & $2 / 58(3.4 \%)$ & $14 / 58(24.1 \%)$ & $39 / 58(67.2 \%)$ & $3 / 58(5.2 \%)$ \\
\hline $1848-2581$ & $0 / 24(0 \%)$ & $4 / 24(16.6 \%)$ & $20 / 24(83.3 \%)$ & $0 / 24(0 \%)$ \\
\hline
\end{tabular}

All results were obtained by injection of dsRNA into eggs. dsRNA fragment names refer to the nucleotides of GenBank sequence AF356647 included in the fragment. Severity of effect was classified as follows. Larvae with mild effects had short legs resulting from reduction and fusion of proximal segments. Moderately affected larvae had reductions in the ventral head and labial appendages in addition to short legs. Severely affected larvae were distinguished from those with moderate effects by the failure of the labial appendages to fuse at the ventral midline. 

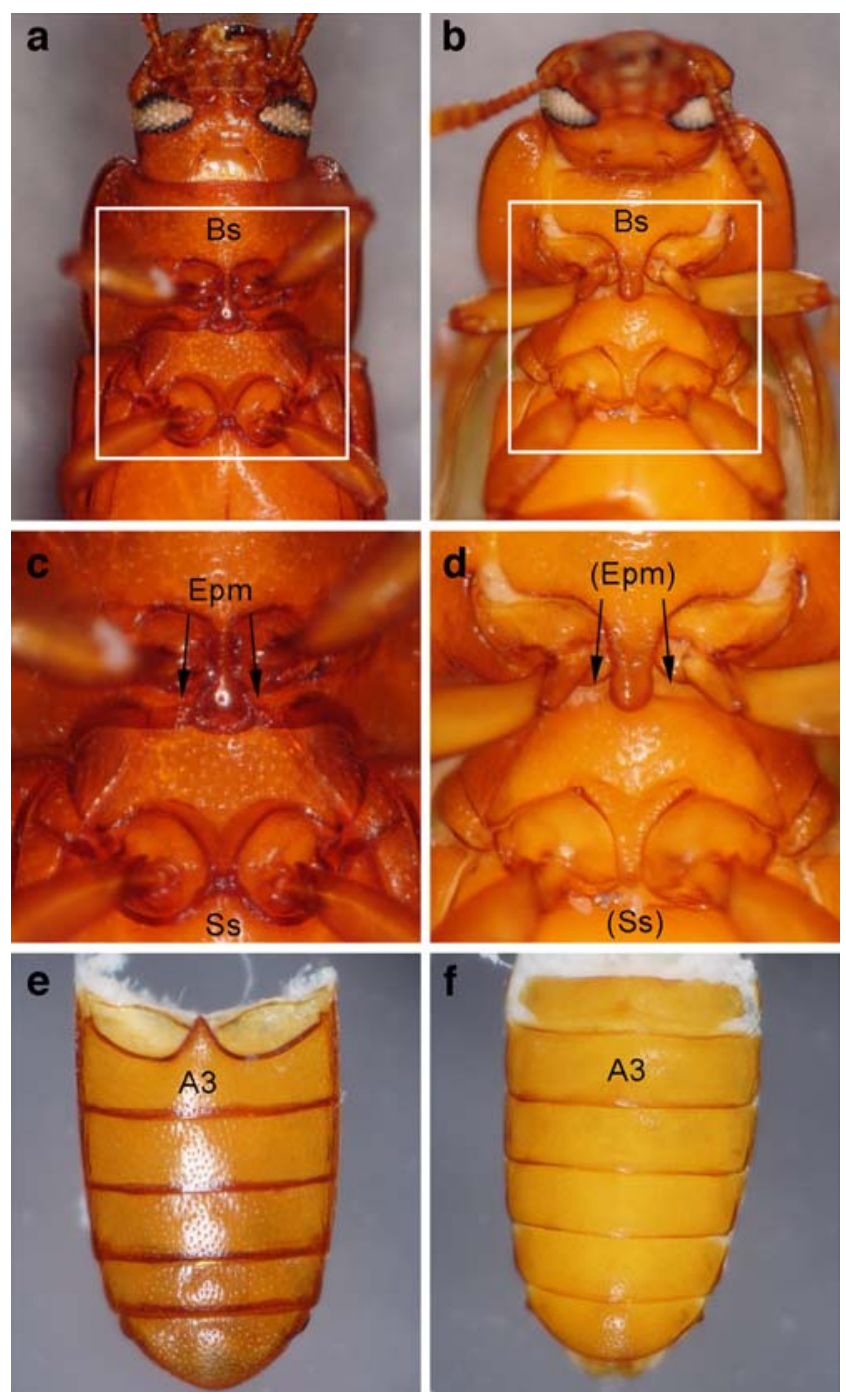

Fig. 6 Adult phenotypes resulting from Tc-tiotsh larval RNAi. All images are ventral views with anterior to the top. a Wild-type adult head and thorax. b Head and thorax of Tc-tiotsh RNAi individual. The T1 basisternum $(B s)$ is smaller than normal. $\mathbf{c}$ Enlarged view of region outlined in A. d Enlarged view of region outlined in B. The T1 epimera $(E p m)$ and the T3 meso-spinasternum $(S s)$ are reduced or missing. e Wild-type adult abdomen. f Abdomen of Tc-tiotsh RNAi adult. The third abdominal segment $(A 3)$ lacks its anterior process, and an additional heavily sclerotized segment is present anterior to $A 3$

extending between the T1 legs) is abnormal. Ventral T2 is not noticeably affected, but the T3 sternite is missing its anterior process (meso-spinasternum) (Fig. 6d). The anterior abdominal segments are also affected by Tc-tiotsh larval RNAi. In wild-type adults, the first and second abdominal segments (A1-A2) form an internal portion of the T3 leg sockets and are only lightly sclerotized, while $\mathrm{A} 3$ is distinguished by a sharply pointed process extending between the T3 legs (Fig. 6e). In Tc-tiotsh RNAi individuals, an additional anterior region (A1 and/or A2) is heavily sclerotized and A3 lacks its anterior process, thus resem- bling the more posterior abdominal segments (A4-A6) (Fig. 6f).

\section{Discussion}

We have identified and cloned the lone member of the $t s h$ gene family from Tribolium castaneum. Our analysis of this gene provides important insights into the evolution of the tsh gene family at the levels of both sequence and function.

\section{Evolution of $t s h$ family genes}

Searches of several non-drosophilid insect genome sequences identified only a single $t s h$ family member in each genome. These results corroborate previous suggestions (based on more limited data) that most insects have only a single tsh-related gene and that this is the ancestral state (Herke et al. 2005; Laugier et al. 2005). A key implication of this model is that the Drosophila genes tio and tsh are the result of a recent duplication event that occurred sometime after the divergence of mosquitoes and flies. Since that duplication, $t s h$ has apparently diverged from the ancestral sequence faster than has tio (although both have diverged more than the Tribolium and Anopheles homo$\operatorname{logs}$ ). More rapid divergence by one member of a recently duplicated gene pair is not unprecedented. For example, the Hox3 paralogs zerknüllt (zen) and bicoid (bcd) are believed to have arisen by duplication late in the dipteran lineage (Brown et al. 2001; Stauber et al. 1999, 2002), but the sequence of Zen is more similar to that of the Hox 3 proteins of other insects than it is to Bcd.

Much of the sequence conservation between Tsh family members is found in the zinc finger regions. However, a few additional conserved motifs are present, particularly at the protein termini. The C-terminal region is well conserved between Tc-TioTsh, Ag-TioTsh, and Tio, but this conserved region is not found in Tsh. The conservation of the Nterminal region is particularly interesting. The Tsh family members for which $\mathrm{N}$-terminal sequence is available fall into two distinct classes (Fig. 2c). Ag-TioTsh and both Drosophila proteins share an extended region of identity that is completely different than the short motif (MPRRKQ) shared by the Tribolium and vertebrate proteins. This pattern of conservation suggests that an ancestral Tsh family member had the MPRRKQ motif, which was replaced with a different motif sometime before the separation of Anopheles and Drosophila within the dipteran lineage. It is not clear what the function of these disparate motifs might be, as the vertebrate Tsh proteins function very similarly to Dm-Tsh when expressed in Drosophila (Manfroid et al. 2004). Perhaps these motifs are important in a particular tissue or at a different stage in development 
than those assayed. As additional insect Tsh family protein sequences become available, it will be possible to more accurately determine the point at which these $\mathrm{N}$-terminal sequences began to diverge.

\section{Evolution of $t$ sh family gene expression}

Although the sequences of other insect $t s h$ family members are most similar to tio, their expression patterns (where known) seem to be more similar to tsh. The embryonic expression patterns of tsh and tio in Drosophila are strikingly different (Laugier et al. 2005). tsh is expressed throughout the ectoderm of trunk segments, while tio is expressed in only a few cells of the trunk ectoderm (as well as in the Malpighian tubules and parts of the CNS and gut). In $t s h$ mutants, however, tio is more broadly expressed in the trunk, indicating that $t s h$ represses tio expression in this region. Likewise, tsh is ectopically expressed in the stomodeal region when tio expression is knocked down by RNAi.

The Tc-tiotsh expression pattern includes similiarities to both the tsh and tio expression patterns. The ectodermal expression in the segmented region of the germband is reminiscent of, although more extensive than, that of $t s h$. On the other hand, Tc-tiotsh is expressed in the Malpighian tubule primordia, similar to tio. The expression of tio near the stomodeum might also correspond to the expression of Tc-tiotsh in the labrum and intercalary segment, although it is difficult to compare expression patterns in the cephalic regions of these insects due to the derived structure of the Drosophila larval head.

It is interesting that the Tc-tiotsh expression pattern approximates the sum of the $t s h$ and tio patterns. This may indicate that the expression pattern of the ancestral gene that gave rise to tsh and tio had an expression pattern similar to Tc-tiotsh and that parts of its expression pattern (and perhaps its function) have been split between its daughters. Such division of labor resulting from complementary loss of particular expression domains or functions is termed subfunctionalization and has been suggested to play a key role in the maintenance of duplicated genes (Force et al. 1999).

Among other insects, expression patterns of tsh family members have been reported only for the firebrat, Thermobia domestica, and the milkweed bug, Oncopeltus fasciatus (Herke et al. 2005; Peterson et al. 1999). In both cases, these genes are expressed in the gnathal, thoracic, and first abdominal segments, with very weak (if any) expression in the remainder of the abdomen. Both insects also show expression in the posterior tip of the embryo (see below). Oncopeltus has additional expression in the procephalon. The leg expression pattern in firebrats has not been described, but Herke et al. (2005) reported that milkweed bugs show expression throughout the legs, which fades from the distal tips later in development.

Interestingly, all of the insect tsh family genes whose expression has been described are expressed in the postabdominal region. However, these domains have been interpreted differently. In firebrats, expression in this region is reported to represent the anlagen of the cerci and the median caudal filament (appendages of the 11th abdominal segment) (Peterson et al. 1999). The posterior expression of $t s h$ has been interpreted as the future anal opening (Fasano et al. 1991), and that of Oncopeltus tiptop is described only as the caudal region (Herke et al. 2005). Our results show that Tc-tiotsh is expressed in the Malpighian tubule primordia. Likewise, tio is expressed in the developing Malpighian tubules (Laugier et al. 2005). Thus, Malpighian tubule expression may have been part of the ancestral expression pattern of this gene family. It is possible that the posterior expression domains in other insects will, upon closer examination, be found to represent the developing Malpighian tubules as well.

\section{Evolution of tsh family function}

Tc-tiotsh is required for the proper development of the proximal portion of developing larval legs. Drosophila larvae lack external appendages, but $t s h$ is expressed in the proximal regions of leg imaginal discs and hypomorphic tsh alleles cause proximal leg defects (Erkner et al. 1999). These results suggest that proximal leg expression and function were probably a feature of a $t s h$-like gene in the ancestor of beetles and flies. In contrast, Herke et al. (2005) reported that knockdown of Oncopeltus tiptop caused transformations of the distal legs to antennae. Based on this observation, they concluded that Oncopeltus tiptop acts as a distal leg selector gene, and suggested that tio or tsh might play a similar role in Drosophila. tsh expression is known to be restricted to the portion of the imaginal disc that will form the body wall and proximal leg segments (Gonzalez-Crespo and Morata 1996). Similarly, Tc-tiotsh is expressed in the proximal region of embryonic legs and leg imaginal discs (Fig. 4d and data not shown). We have also examined the expression of tio in Drosophila leg imaginal discs and found that expression is restricted to the body wall and proximal portion of the developing leg. Thus, it is highly unlikely that the "tiptop-like" distal leg-specifying function suggested by Herke et al. (2005) is performed by Tio, either alone or in combination with Tsh.

We cannot rule out additional, internal functions for TcTioTsh. In particular, the expression of Tc-tiotsh in the Malpighian tubules is intriguing, as Drosophila tio (and possibly other homologs) show similar expression.

The striking effects of larval Tc-tiotsh RNAi would not have been predicted based on Drosophila experiments, as 
relatively few adult effects of LOF tsh mutations have been reported. Other than the proximal leg defects that have been discussed above, the only other adult phenotypes reported are defects in wing posture (Soanes et al. 2001) and in structures that develop from the eye-antennal disc, namely, the eyes (Singh et al. 2002) and the maxillary palps (Bhojwani et al. 1997). This dearth of adult phenotypes could be a consequence of the pre-adult lethality of many tsh allele combinations. However, it might also be due to functional redundancy with tio. We examined tio expression in Drosophila imaginal discs and found that it is indeed expressed in a pattern very similar to that of $t s h$ (data not shown). Thus, loss of both tio and tsh during Drosophila imaginal development might produce a more severe phenotype.

The principal question prompting our studies of the Tribolium Tc-tiotsh gene was whether the trunk-specifying function reported for tsh in Drosophila was conserved in other insects. However, insights from our work and other recent reports compel us to first reexamine whether tsh itself is really a trunk-specifying gene. The key observations, which originally led to this conclusion, were the presence of head-like sclerites in embryos lacking tsh, and the positive correlation between expression of $t s h$ and the presence of denticle belts (a trunk-specific feature) (de Zulueta et al. 1994; Fasano et al. 1991; Roder et al. 1992). The later discovery that tsh and disco form mutually exclusive domains in the trunk and head, respectively, seemed to support this view (Robertson et al. 2004). Below we synthesize recent studies that may lead to new interpretations of these observations.

A number of mutant phenotypes in Drosophila, including those of $t s h$ and some Hox genes, feature small patches of sclerotic tissue. The previous interpretation of such patches as ectopic head tissue has been called into question. Mace et al. (2005) showed that the sclerites that form in a segment lacking Hox gene expression are correlated with breaches in epidermal integrity and the resulting activation of a wound response pathway. If in fact, the sclerotized cuticle seen in tsh mutants is scar tissue rather than head cuticle, the interpretation of $t s h$ as a trunk-specifying gene would be less convincing, but still possible.

The case for tsh acting as a trunk specifier was apparently strengthened when Robertson et al. (2004) proposed the attractive hypothesis that a system of zinc finger proteins acted in conjunction with the Hox genes to specify body regions. Within this system, disco specified gnathal segments and tsh specified trunk segments. However, the same laboratory recently reported that the conserved role of disco is to specify the medial portion of appendages, and suggested that the boundary between disco and $t s h$ expression is actually an artifact of maggot morphology (Patel et al. 2007). That is, the reduced
Drosophila larval head might lack proximal, tsh-expressing tissue (body wall) while retaining medial appendage tissue, whereas the trunk retains $t s h$-expressing tissue but has lost most appendage tissue. Taking this scenario a step further, ectopic expression of tsh might "restore" the body wall tissue of the head, creating larger, more complete segments. As the genes controlling segment formation also control denticle belt patterning, these enlarged head segments would have denticle belts as well. This interpretation is consistent with the observation that the additional denticle belts produced by ectopic $t s h$ expression are interspersed with head-specific structures (de Zulueta et al. 1994). When considered in this light, perhaps $t s h$ is no more a trunk identity gene than are other genes involved in segment formation and denticle patterning such as wingless, rhomboid, or shavenbaby. On the other hand, tsh does seem to provide the spatial information that limits full segment formation to the trunk, and thus in Drosophila could be considered a trunk-specifying gene.

In contrast, Tc-tiotsh does not appear to act in any way as a trunk identity gene. This is not to say that $T c$-tiotsh has no role in the development of trunk segments, but rather that we find no evidence that it specifies trunk vs head fates. In embryos depleted of Tc-tiotsh the ventral portions of the labial and thoracic segments are reduced, but not visibly transformed. Interestingly, some of the labial abnormalities observed in Tc-tiotsh RNAi larvae are reminiscent of those produced by loss of function alleles of prothoraxless (TcAntp) (Shippy, unpublished data). Knockdown of Tc-tiotsh during imaginal development produces adults with patterning defects in the thoracic and abdominal sclerites, including possible transformations of anterior abdominal sclerites to a more posterior identity. These phenotypes, together with the reduction in Tc-tiotsh expression seen in $\mathrm{Df}(\mathrm{HOMC})$ embryos, suggest that $T c$-tiotsh is regulated to some extent by Hox genes and may carry out a subset of the functions of these genes in both the head and the trunk.

Our studies of Tc-tiotsh indicate that trunk specification is not a universal function of tsh family members. It is possible that this function has been lost in Tribolium. However, expression pattern data from other insects and functional analysis in Oncopeltus argues against this conclusion. A more likely scenario is that tsh family members acquired a role in defining trunk at some point after the divergence of the coleopteran and dipteran lineages. Analysis of $t s h$ function in additional holometabolous insects should help pinpoint when this event occurred.

Acknowledgments We thank Marcé Lorenzen for performing some of the embryonic RNAi injections, Sue Haas for helping characterize the embryonic RNAi phenotypes, and Deane Lehmann and Michelle Coleman for technical assistance. We are also grateful to members of the Denell and Brown laboratories for helpful discussions and to Kent 
Hampton of the Kansas Agricultural Experiment Station Scanning Electron Microscope Laboratory in the Department of Entomology at Kansas State University for expert assistance with SEMs. This work was supported by grants from the National Science Foundation (IOB0321882) (RED, SJB and TDS), the National Institutes of Health (HD29594) (SJB, RED and TDS), the Japanese Society for the Promotion of Science (YT), and the Terry C. Johnson Center for Basic Cancer Research at Kansas State University.

Open Access This article is distributed under the terms of the Creative Commons Attribution Noncommercial License which permits any noncommercial use, distribution, and reproduction in any medium, provided the original author(s) and source are credited.

\section{References}

Bhojwani J, Shashidhara LS, Sinha P (1997) Requirement of teashirt (tsh) function during cell fate specification in developing head structures in Drosophila. Dev Genes Evol 207:137-146

Brown SJ, Hilgenfeld RB, Denell RE (1994a) The beetle Tribolium castaneum has a fushi tarazu homolog expressed in stripes during segmentation. Proc Natl Acad Sci USA 91:12922-12926

Brown SJ, Patel NH, Denell RE (1994b) Embryonic expression of the single Tribolium engrailed homolog. Dev Genet 15:7-18

Brown SJ, Mahaffey JP, Lorenzen MD, Denell RE, Mahaffey JW (1999) Using RNAi to investigate orthologous homeotic gene function during development of distantly related insects. Evol Dev 1:11-15

Brown S, Fellers J, Shippy T, Denell R, Stauber M, Schmidt-Ott U (2001) A strategy for mapping bicoid on the phylogenetic tree. Curr Biol 11:R43-R44

Brown SJ, Shippy TD, Beeman RW, Denell RE (2002) Tribolium Hox genes repress antennal development in the gnathos and trunk. Mol Phylogenet Evol 24:384-387

Caubit X, Core N, Boned A, Kerridge S, Djabali M, Fasano L (2000) Vertebrate orthologues of the Drosophila region-specific patterning gene teashirt. Mech Dev 91:445-448

Curtis CD, Brisson JA, DeCamillis MA, Shippy TD, Brown SJ, Denell RE (2001) Molecular characterization of Cephalothorax, the Tribolium ortholog of Sex combs reduced. Genesis 30:12-20

de Zulueta P, Alexandre E, Jacq B, Kerridge S (1994) Homeotic complex and teashirt genes co-operate to establish trunk segmental identities in Drosophila. Development 120:2287-2296

Drosophila 12 Genomes Consortium (2007) Evolution of genes and genomes on the Drosophila phylogeny. Nature 450:203-218

El-Kifl AH (1953) Morphology of the adult Tribolium confusum Duv. and its differentiation from Tribolium (Stene) castaneum Herbst. Bull Soc Fouad 1er Entomol 37:173-249

Erkner A, Gallet A, Angelats C, Fasano L, Kerridge S (1999) The role of Teashirt in proximal leg development in Drosophila: ectopic Teashirt expression reveals different cell behaviours in ventral and dorsal domains. Dev Biol 215:221-232

Fasano L, Roder L, Core N, Alexandre E, Vola C, Jacq B, Kerridge S (1991) The gene teashirt is required for the development of Drosophila embryonic trunk segments and encodes a protein with widely spaced zinc finger motifs. Cell 64:63-79

Force A, Lynch M, Pickett FB, Amores A, Yan Y-L, Postlethwait J (1999) Preservation of duplicate genes by complementary, degenerative mutations. Genetics 151:1531-1545

Gonzalez-Crespo S, Morata G (1996) Genetic evidence for the subdivision of the arthropod limb into coxopodite and telopodite. Development 122:3921-3928
Herke SW, Serio NV, Rogers BT (2005) Functional analyses of tiptop and antennapedia in the embryonic development of Oncopeltus fasciatus suggests an evolutionary pathway from ground state to insect legs. Development 132:27-34

Holt RA, Subramanian GM, Halpern A, Sutton GG, Charlab R et al (2002) The genome sequence of the malaria mosquito Anopheles gambiae. Science 298:129-149

Honeybee Genome Sequence Consortium (2006) Insights into social insects from the genome of the honeybee Apis mellifera. Nature 443:931-949

Kennerdell JR, Carthew RW (2000) Heritable gene silencing in Drosophila using double-stranded RNA. Nat Biotechnol 18:896898

Kumar S, Tamura K, Nei M (2004) MEGA3: integrated software for molecular evolutionary genetics analysis and sequence alignment. Brief Bioinform 5:150-163

Laugier E, Yang Z, Fasano L, Kerridge S, Vola C (2005) A critical role of teashirt for patterning the ventral epidermis is masked by ectopic expression of tiptop, a paralog of teashirt in Drosophila. Dev Biol 283:446-458

Mace KA, Pearson JC, McGinnis W (2005) An epidermal barrier wound repair pathway in Drosophila is mediated by grainy head. Science 308:381-385

Manfroid I, Caubit X, Kerridge S, Fasano L (2004) Three putative murine Teashirt orthologues specify trunk structures in Drosophila in the same way as the Drosophila teashirt gene. Development 131:1065-1073

Martindale MQ (2005) The evolution of metazoan axial properties. Nat Rev Genet 6:917-927

Nie W, Stronach B, Panganiban G, Shippy T, Brown S, Denell R (2001) Molecular characterization of Tclabial and the 3' end of the Tribolium homeotic complex. Dev Genes Evol 211:244-251

Ochman H, Gerber AS, Hartl DL (1988) Genetic applications of an inverse polymerase chain reaction. Genetics 120:621-623

Patel NH, Kornberg TB, Goodman CS (1989) Expression of engrailed during segmentation in grasshopper and crayfish. Development 107:201-212

Patel NH, Condron BG, Zinn K (1994) Pair-rule expression patterns of even-skipped are found in both short- and long-germ beetles. Nature 367:429-434

Patel M, Farzana L, Robertson LK, Hutchinson J, Grubbs N, Shepherd MN, Mahaffey JW (2007) The appendage role of insect disco genes and possible implications on the evolution of the maggot larval form. Dev Biol 309:56-69

Peterson MD, Rogers BT, Popadic A, Kaufman TC (1999) The embryonic expression pattern of labial, posterior homeotic complex genes and the teashirt homologue in an apterygote insect. Dev Genes Evol 209:77-90

Prpic NM, Wigand B, Damen WG, Klingler M (2001) Expression of dachshund in wild-type and distal-less mutant Tribolium corroborates serial homologies in insect appendages. Dev Genes Evol 211:467-477

Putnam NH, Srivastava M, Hellsten U, Dirks B, Chapman J, Salamov A, Terry A, Shapiro H, Lindquist E, Kapitonov VV, Jurka J, Genikhovich G, Grigoriev IV, Lucas SM, Steele RE, Finnerty JR, Technau U, Martindale MQ, Rokhsar DS (2007) Sea anemone genome reveals ancestral eumetazoan gene repertoire and genomic organization. Science 317:86-94

Richards S, Liu Y, Bettencourt BR, Hradecky P, Letovsky S, Nielsen R, Thornton K, Hubisz MJ, Chen R, Meisel RP, Couronne O, Hua S, Smith MA, Zhang P, Liu J, Bussemaker HJ, van Batenburg MF, Howells SL, Scherer SE, Sodergren E, Matthews BB, Crosby MA, Schroeder AJ, Ortiz-Barrientos D, Rives CM, Metzker ML, Muzny DM, Scott G, Steffen D, Wheeler DA, Worley KC, Havlak P, Durbin KJ, Egan A, Gill R, Hume J, Morgan MB, Miner G, Hamilton C, Huang Y, Waldron L, 
Verduzco D, Clerc-Blankenburg KP, Dubchak I, Noor MA, Anderson W, White KP, Clark AG, Schaeffer SW, Gelbart W, Weinstock GM, Gibbs RA (2005) Comparative genome sequencing of Drosophila pseudoobscura: chromosomal, gene, and ciselement evolution. Genome Res 15:1-18

Robertson LK, Bowling DB, Mahaffey JP, Imiolczyk B, Mahaffey JW (2004) An interactive network of zinc-finger proteins contributes to regionalization of the Drosophila embryo and establishes the domains of HOM-C protein function. Development 131:27812789

Roder L, Vola C, Kerridge S (1992) The role of the teashirt gene in trunk segmental identity in Drosophila. Development 115:10171033

Sato T, Hayes PH, Denell RE (1985) Homeosis in Drosophila: roles and spatial patterns of expression of the Antennapedia and Sex combs reduced loci in embryogenesis. Dev Biol 111:171-192

Singh A, Kango-Singh M, Sun YH (2002) Eye suppression, a novel function of teashirt, requires Wingless signaling. Development 129:4271-4280

Soanes KH, MacKay JO, Core N, Heslip T, Kerridge S, Bell JB (2001) Identification of a regulatory allele of teashirt (tsh) in
Drosophila melanogaster that affects wing hinge development. An adult-specific tsh enhancer in Drosophila. Mech Dev 105:145-151

Stauber M, Jackle H, Schmidt-Ott U (1999) The anterior determinant bicoid of Drosophila is a derived Hox class 3 gene. Proc Natl Acad Sci USA 96:3786-3789

Stauber M, Prell A, Schmidt-Ott U (2002) A single Hox3 gene with composite bicoid and zerknullt expression characteristics in nonCyclorrhaphan flies. Proc Natl Acad Sci USA 99:274-279

Struhl G (1983) Role of the esc+gene product in ensuring the selective expression of segment-specific homeotic genes in Drosophila. J Embryol Exp Morphol 76:297-331

Stuart JJ, Brown SJ, Beeman RW, Denell RE (1991) A deficiency of the homeotic complex of the beetle Tribolium. Nature 350:72-74

Tomoyasu Y, Denell RE (2004) Larval RNAi in Tribolium (Coleoptera) for analyzing adult development. Dev Genes Evol 214:575-578

Tomoyasu Y, Wheeler SR, Denell RE (2005) Ultrabithorax is required for membranous wing identity in the beetle Tribolium castaneum. Nature 433:643-647

Tribolium Genome Sequence Consortium (2008) The genome of the model beetle and pest Tribolium castaneum. Nature (in press) 